\title{
Power-Driven Design Partitioning
}

\author{
Rajarshi Mukherjee, Seda Ogrenci Memik \\ Electrical and Computer Engineering, Northwestern University \\ \{rajarshi, seda\}@ece.northwestern.edu
}

\begin{abstract}
In order to enable efficient integration of FPGAs into cost effective and reliable high-performance systems as well potentially into low power mobile systems, their power efficiency needs to be improved. In this paper, we propose a power management scheme for FPGAs centered on a power-driven partitioning technique. Our power-driven partitioner creates clusters within a design such that within individual clusters, power consumption can be improved via voltage scaling. We tested the effectiveness of our approach on a set of LUT-level benchmark netlists. Further we did constrained placement of the clusters into predefined $\mathrm{V}_{\mathrm{dd}}^{\text {high }}$ and $\mathrm{V}_{\mathrm{dd}}^{\text {low }}$ regions for a single FPGA. Average savings in power consumption with our approach is $48 \%$ whereas penalty in channel width and wire length due to constrained placement is $23 \%$ and $26 \%$ respectively.
\end{abstract}

\section{Introduction}

Despite exponential improvements in logic density and performance, energy efficiency of the FPGA technology did not keep up. As heat dissipation becomes an increasingly important concern for wired systems, power consumption of FPGAs needs to match more stringent standards in order to ensure the performance goals and reliability. Similarly, inefficiency in power consumption poses a major obstacle to inclusion of FPGAs in many emerging low power mobile systems.

In this paper, we present a high-level power management methodology for FPGAs. We propose a power-driven partitioning technique that identifies partitions in a design, which can be implemented using lower supply voltage levels. Through voltage scaling we allow longer delay for parts of a design while the overall latency of the design is unchanged. In every design, based on the overall timing constraint, there is a set of critical nodes/operations/gates, while the remainder of the design is non-critical. Hence, it is possible to identify the inherent time slack possessed by individual building blocks in a design. We analyze this design metric and systematically exploit potential relaxation in timing constraints in order to create opportunities for voltage scaling. Our techniques can be utilized for various FPGA-based systems. In multi-FPGA systems applications are partitioned among several devices. Using our partitioning technique voltage scaling can be applied at chip level, using different supply voltages for different devices. While mapping a design onto a single FPGA chip, the design is partitioned such that portions identified by our partitioning technique can be placed within voltage islands at different $V_{d d}$ levels on the same chip. Finally, for dynamically reconfigurable systems, the voltage supply level can be dynamically adjusted and our partitioning technique can be used to create configuration contexts such that lowering of the supply voltage is feasible for some of the partitions. 
Our specific contributions in this paper are as follows:

- We propose a partitioning algorithm that effectively exploits relaxable timing constraints within a design to trade-off delay against lower supply voltage levels,

- We present experimental results using LUT-level netlists to demonstrate the improvement in power using our proposed technique, and

- We present an experimental evaluation of the impact of creating voltage islands on the physical design stages.

The rest of the paper is organized as follows. In Section 2 we present an overview of related work. Section 3 presents the problem formulation and our algorithm. Our experimental setup and results will be presented in Section 4. We conclude with a summary in Section 5 .

\section{Related Work}

In the past, various proposed synthesis techniques addressed the problem of power optimization by improving metrics such as switching activity of a schedule or binding, total used logic resources, interconnect, etc. to generate the most efficient circuit in terms of power consumption [1], [2], [3], [4], [5]. In our approach we are addressing a different power optimization paradigm, namely voltage scaling. Our work is complementary to the above-mentioned techniques.

Partitioning has been studied for multi-FPGA systems and for dynamically reconfigurable systems [6], [7], [8], [9]. The main objectives for optimization have been traditionally cut cost, i.e., the number of connections between partitions, and the number of partitions. In this work, we propose a partitioning scheme that addresses a new objective, namely availability of time slack in a partition. We investigate the potential impact of partitioning on power. Depending on the particular application of our technique, we take other partitioning objectives into account as well.

Voltage scaling is a well-known tool for improving energy efficiency of electronic systems. It has found applications for a wide variety of circuit technologies and design styles including microprocessors, ASICs and real time embedded systems [10], [11], [12], [13]. In this work, we make use of this general optimization technique and investigate its application on FPGA-based systems. Investigation of circuit level issues to implement voltage scaling is beyond the scope of this paper. However, the feasibility of implementing the necessary hardware for voltage scaling is evident considering the successful implementations in other technologies. In addition, Chen et al. reported recent results on the feasibility of dual supply voltage FPGA fabrics [14], [15].

\section{Power Management Using Voltage Scaling}

In this section, we will formulate our power management problem and discuss two applications of our proposed technique. Next, we will describe our power-driven partitioning algorithm in detail. 


\subsection{Problem Formulation}

We assume that a LUT-level netlist is represented with a Directed Acyclic Graph (DAG). The longest path from any of the primary inputs to any of the primary outputs defines the longest combinational path, i.e., the critical path in the design. Logic blocks that reside on the critical path are called critical nodes. The rest is referred to as non-critical nodes. Taking the length of the critical path as our timing constraint and assuming that all input signals arrive at the same time, we can assign arrival and required times for each node. The difference between the required time and the arrival time of a node is called time slack. This entity will be equal to zero for critical nodes, while it takes a positive value for non-critical nodes. Note that we are estimating the time slack of each LUT at a high level. Naturally, the timing behavior of a design will highly depend on the net delays, hence, on placement and routing. An accurate estimation of interconnect delay cannot be made before physical synthesis. Being aware of this fact, we will evaluate the impact of placement and routing. Details of this study will be presented in Section 4.3. We would like to stress once again that time slack as defined above is the best estimation we can have at this early stage of the design flow to identify the non-critical portions of a design.

Our power management technique relies on the observation that the slack possessed by individual nodes can be used as a guide to create partitions within which all nodes would maintain a certain level of timing freedom, which in turn can be exploited through scaling the voltage supply fed into that partition. It is well known that the dynamic power reduces by the square of the supply voltage $\left(\mathrm{P} \sim \mathrm{C}_{\text {load }} \mathrm{V}_{\text {dd }}{ }^{2} \mathrm{f}_{\text {switch }}\right)$, and the delay increases linearly $\left(\mathrm{D} \sim \mathrm{C}_{\text {load }} / \mathrm{V}_{\mathrm{dd}}\right)$ as the supply voltage is decreased. Hence, it is possible to perform a tradeoff between power consumption and performance by changing the supply voltage.

Our aim is to identify partitions in a design, such that the total power consumption is minimized while resource constraints associated with the partitioning problem are satisfied. For a given partition, the length of the longest path and the amount of time slack available along that path will be used to compute the voltage scaling within a partition. The voltage-scaling factor (Sc_Fac) is then defined as

$$
S c_{-} F a c=\frac{\text { longest_path_length_in_partition }}{\text { longest_path_length_in_partition }+ \text { slack }}
$$

Our partitioning scheme can find applications at various levels. Next we differentiate between two cases and are shown in Figure 1.

\subsubsection{Chip-level Voltage Scaling for Multi-FPGA Systems}

Many FPGA-based hardware acceleration systems employ multiple FPGAs. Partitioning is frequently used to map a large design onto several FPGA devices. Such systems are generally wired; hence, they do not operate under a tight power budget. However, heat dissipation is becoming a growing concern. Overhead due to cooling systems can be reduced through effective power management. More importantly, reliability issues arising due to excessive heat dissipation require closer attention as the FPGA manufacturing technology reached submicron levels. For multiFPGA systems we apply our partitioning technique to create partitions such that some 
of those partitions can be assigned to a device operating at a lower supply voltage level.

\subsubsection{Localized Voltage Scaling for Single FPGA Systems}

A design flow targeting a single FPGA device can also benefit from our powerdriven partitioning technique. In this case, by embedding voltage islands on a chip we can enable different parts of a design to operate at different voltage levels. Generating these voltage islands on a single FPGA will incur certain hardware costs. Considering the overhead of creating voltage islands -additional circuitry for voltage scaling, level conversion, etc. the number and layout of different voltage islands can be constrained. We believe that the benefits of voltage scaling will justify the additional hardware cost in the next generation FPGA architectures. We will elaborate more on this issue as we present our results in Section 4.

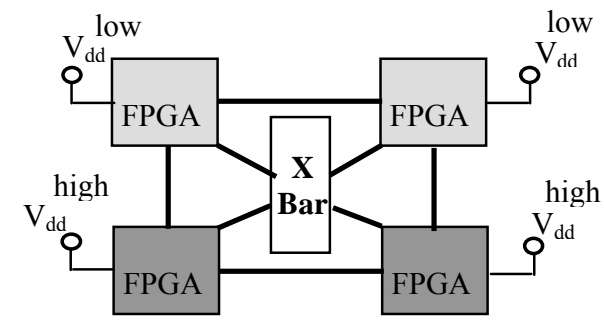

(a)

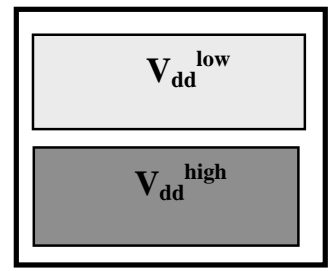

(b)

Figure 1. Application of voltage scaling in two different scenarios. (a) Multi-FPGA system. (b) Single FPGA containing voltage islands.

\subsection{Power-Driven Partitioning Algorithm}

Our algorithm takes in a LUT - level netlist. The input buffer connected to the input port $(I B U F)$ is considered a primary input and output buffer connected to the output port $(O B U F)$ is considered a primary output. We assume that each LUT has a delay of 1 unit when operating at full supply voltage level.

Our algorithm tries to identify clusters of nodes along a path, which can share the time slack available along that path. The reasons why we consider only nodes along a path are twofold. First, since nodes along a path have data dependencies, they are likely to be placed in the same partition (or be placed close physically in the case of single FPGA systems) even without voltage scaling considerations. Second, the slack values on the nodes along a path are usually close to each other. Clearly, a good power-driven partitioning algorithm clusters nodes with similar slack values together.

Our algorithm first finds the slack values on the nodes, forms an initial set of clusters assuming the availability of arbitrary scaling factors. Then, in a refinement phase the voltage scaling factors of the clusters are adjusted according to the available supply voltage levels in the target hardware.

The slack at each node is calculated by computing the difference of required and arrival times of the signals by sorting the nodes in topological order. The algorithm takes as input the feasible scale factor Sc_Fac - the minimum amount by which volt- 
age can be scaled. Then, it selects the nodes in topological order thereby selecting nodes from the input level and going towards the output. If the node has zero slack it is added to the non-scaled partition. After choosing a non-zero slack node $v$, which is not already added to any partition, it is checked whether

$$
S_{-} c_{-} \text {Fac }>\frac{\text { longest_path_in_partition }}{\text { longest_path_in_partition + slack }}
$$

If this condition is satisfied, then a new cluster is created and the algorithm tries to grow the cluster. (As we add the first node to a partition, the delay of the longest path in the partition is equal to the delay of the node itself.) Iteratively, minimum slack fanout nodes are selected (not already in another cluster) and added to the cluster if it is feasible to add a new node and the slack of the path is updated as the minimum of the slacks of the nodes in the path. Let us call this entity slack ${ }^{\text {Path }}$. Similarly, the length of the longest path in the partition is updated.

Once we stop adding any more nodes to a cluster $c_{i}$, we will have the following information about this cluster: $\boldsymbol{M}_{\boldsymbol{i}}$ : number of nodes along the longest path in $\boldsymbol{C}_{\boldsymbol{i}}, \boldsymbol{L}_{\boldsymbol{i}}$ : length of the longest path in $c_{i}$, slack ${ }^{\text {Path }}$ : available slack along the longest path in $c_{i}$. (Without violating any overall timing constraints of the circuit, we can slow down the longest path in this cluster by slack ${ }^{\text {Path }}$ time units.), node_delay: amount of time by which each individual node in $c_{i}$ can be slowed down. We can formally express node_delay as follows:

$$
\text { node_delay }=\frac{\text { slack }^{\text {Path }}}{M_{i}}
$$

Since the same voltage-scaling factor will be applied to all nodes within a partition, the slowdown of each node will be same. The voltage supply for this cluster can be scaled down by the factor of

$$
\text { scale }^{\text {cluster }_{i}}=\frac{L_{i}}{L_{i}+\text { slack }^{\text {Path }}}
$$

Iteratively, clusters are created until all nodes are assigned to a cluster.

\subsubsection{Post processing of Clusters}

After creating an initial set of clusters our next goal is to assign these clusters into voltage scaled FPGAs or individual voltage islands on a single chip. The circuitry employed for scaling will have a pre-defined sensitivity. In other words, the incremental steps by which we can adjust the voltage level are quantized, e.g. voltage scaling can be within a range of 0.64 Volts in 8 steps of 0.08 Volts. First, we perform a pass over all clusters and round up their voltage levels to the nearest feasible level.

If the number of clusters in the initial partition is less than or equal to the number of FPGA devices in the system then the assignment is straightforward. For the single FPGA case, we will identify two distinct voltage levels: $\mathrm{V}_{\mathrm{dd}}^{\text {high }}$ and $\mathrm{V}_{\mathrm{dd}}$ low . Hence, each cluster will be merged to either one of these two levels. For the multiFPGA case, we can allow more voltage levels since one voltage regulator will serve one individual chip. We developed two heuristics to achieve the finalized partitioning of the netlist into $N$ voltage scaled partitions ( $N=2$ for single FPGA scenario). We refer to these schemes as Delay Based Merging and Connectivity Based Merging. 
Delay Based Merging tries to satisfy the resource constraint while searching for the best possible merging in terms of power gain. Essentially, this method tries to group nodes with the most similar slack values together in the same merged cluster. Connectivity Based Merging puts higher emphasis on reducing the cut cost of the final partitioning result. It achieves this by merging clusters with highest number of mutual connections together. We omitted the details of these post-processing heuristics and present only the results of Connectivity Based Merging due to space considerations. After completing the partitioning phase, the total power consumption now becomes $\mathrm{P}_{\text {scale. }}$. If the total power consumption without any voltage scaling was $\mathrm{P}$, the ratio is given by

$$
\frac{P_{\text {scale }}}{P}=\frac{\left(V_{d d}^{\text {scale }}\right)^{2} \times k+(n-k)}{n}
$$

where, $n$ is the total number of nodes in the circuit and $k$ is the number of nodes in the voltage scaled partition. For an $r$-way partition into $r$ FPGA devices, we take the same approach. Assume that the partition sizes are $k_{1}, k_{2}, k_{3}, \ldots k_{r}$. The ratio of the scaled power consumption to the non-scaled power consumption is given by

$$
\frac{P_{\text {scale }}}{P}=\frac{\left(V_{d d}^{\text {scale }_{1}}\right)^{2} \times k_{1}+\left(V_{d d}^{\text {scale }_{2}}\right)^{2} \times k_{2}+\ldots+\left(V_{d d}^{\text {scale }_{r}}\right)^{2} \times k_{r}}{n}
$$

\section{Experiments}

We present our experimental results in this section. First, we summarize our experimental setup and the parameters we used. Then, we report achieved reduction in total power consumption on a collection of benchmarks.

\subsection{Experimental Setup and Parameters}

The experiments are formed on a set of MCNC combinational benchmarks. Further we also tested with synthetic benchmarks of 1000, 5000, 10000 and 15000 nodes. Table 1 summarizes relevant characteristics: number of nodes, edges and critical nodes - the number of nodes with zero slack in the DAG representation of each MCNC benchmark. In Table 2 we show the average values of the same for synthetic benchmarks. It is to be noted that though the results are presented for combinational circuits, the algorithm is equally applicable to sequential circuits where its combinational block can be partitioned into voltage clusters.

The LUT level netlist for MCNC benchmarks were in .net format. The powerdriven partitioner reads in an .edif or .net file and produces a partition for $N$ FPGAs in a multi-FPGA system or $N=2$ voltage islands on a single FPGA. The initial delay of each LUT is assumed to be 1 unit. We further assumed that voltage scaling is done in increments of 0.06 Volts and scale factor is 0.62 . Finally, we assume that for the multi-FPGA scenario, all FPGA devices in the system are identical, i.e., all have the same capacity. Our techniques are independent of the actual values of these parameters; hence, they can operate under different assumptions. 


\subsection{Results: Power Driven Partitioning}

After creating clusters we perform an initial refining, where we round off voltage scaling values according to the smallest scaling step. Next we perform our post processing heuristics of Delay Based Merging and Connectivity Based Merging. In both the merging we generate $\mathrm{N}$-voltage clusters each of roughly the same size. We observe that while we obtain better power improvement using Delay Based Merging the cut cost is consistently inferior to the Connectivity Based Merging. Symmetrically, Connectivity Based Merging always yields better-cut cost at the expense of reduced power improvement. Hence, the possible trade-off between the cut cost objective and power improvement is evident. Table 1 and Table 2 shows the results of only connectivity based merging. We report the cut cost, i.e., the number of inter partition connections as well as the percentage power improvement obtained. For MCNC benchmarks, the average power improves $13.4 \%$ for 2 -way to $14.7 \%$ for 8 way whereas the average cut cost increases from 2389.5 for 2-way to 2530.9 for 8 -way. For Synthetic benchmarks the average power improvement is $27.45 \%$ for 2 -way and $48.2 \%$ for 8 way.

Table 1. Partitioning Results for MCNC benchmarks

\begin{tabular}{|c|r|r|r|r|r|r|r|r|r|}
\cline { 5 - 10 } \multicolumn{4}{c|}{} & \multicolumn{2}{c|}{ 2-way } & \multicolumn{2}{c|}{4 way } & \multicolumn{2}{c|}{ 8-way } \\
\hline MCNC & Nodes & Edges & Crit Nodes & Cost & \%lmp & Cost & $\%$ Imp & Cost & $\%$ Imp \\
\hline Alu4 & 1522 & 5401 & 1180 & 1467 & 13 & 1483 & 13 & 1556 & 17 \\
\hline apex2 & 1878 & 6690 & 1453 & 2158 & 13 & 2190 & 14 & 2335 & 14 \\
\hline apex4 & 1262 & 4461 & 1169 & 440 & 6 & 440 & 6 & 440 & 6 \\
\hline des & 1591 & 5863 & 1326 & 1851 & 18 & 1888 & 18 & 1890 & 19 \\
\hline ex1010 & 4598 & 16069 & 3557 & 5660 & 13 & 5748 & 13 & 5868 & 13 \\
\hline ex5p & 1064 & 3940 & 941 & 663 & 9 & 663 & 9 & 663 & 9 \\
\hline misex3 & 1397 & 4955 & 992 & 1405 & 19 & 1501 & 22 & 1576 & 22 \\
\hline pdc & 4575 & 17154 & 3179 & 4901 & 13 & 5141 & 14 & 5315 & 14 \\
\hline seq & 1750 & 6159 & 936 & 1685 & 21 & 1804 & 21 & 1832 & 21 \\
\hline spla & 3960 & 13763 & 2919 & 3665 & 9 & 3803 & 9 & 3834 & 12 \\
\hline Maximum & & & 5660 & 21 & 5748 & 22 & 5868 & 22 \\
\hline Average & & & 2389.5 & 13.4 & 2466.1 & 13.9 & 2530.9 & 14.7 \\
\hline
\end{tabular}

Table 2. Partitioning Results for synthetic benchmarks (average)

\begin{tabular}{|l|c|c|c|c|c|c|c|r|}
\hline \multicolumn{2}{|l|}{ Synthetic } & \multicolumn{3}{c|}{ 2-way } & \multicolumn{2}{c|}{ 4 way } & \multicolumn{2}{c|}{ 8-way } \\
\hline Nodes & Edges & Crit Nodes & Cost & $\%$ Imp & Cost & $\%$ Imp & Cost & $\%$ Imp \\
\hline 1000 & 1751 & 129 & 360 & 27.2 & 794 & 39.4 & 941 & 47.6 \\
\hline 5000 & 8323 & 209 & 588 & 29.6 & 1392 & 41.8 & 1662 & 50.2 \\
\hline 10000 & 16270 & 276 & 775 & 27.8 & 1962 & 41.6 & 2357 & 48.4 \\
\hline 15000 & 24220 & 359 & 1023 & 25.2 & 2578 & 37.6 & 3087 & 46.6 \\
\hline Maximum & & 1023 & 29.6 & 2578.2 & 41.8 & 3087 & 50.2 \\
\hline Average & & 686.45 & 27.45 & 1681.45 & 40.1 & 2011.8 & 48.2 \\
\hline
\end{tabular}




\subsection{Results: Using Power Driven Partitioning in Physical Design}

We now present the results of the constrained placement of the clusters identified by our algorithm onto for voltage islands supplied by different $\mathrm{V}_{\mathrm{dd}} \mathrm{S}$ on a single FPGA. We propose to use four quadrants as four voltage islands, each of which can be potentially supplied by either $\mathrm{V}_{\mathrm{dd}}^{\text {high }}$ (say $1.3 \mathrm{~V}$ ) or $\mathrm{V}_{\mathrm{dd}}^{\text {low }}$ (say $0.8 \mathrm{~V}$ ) rather than having each individual logic block being $\mathrm{V}_{\mathrm{dd}}$ programmable. The location voltage islands is an architectural parameter. Figure 2 shows the 3 possible configurations. We assume that it is possible to have level converters along the borders of the quadrants. FPGA having dimensions $\mathrm{D}_{\mathrm{X}}, \mathrm{D}_{\mathrm{Y}}$ has total $\left(D_{X} \times D_{Y}\right)$ logic blocks. Our placement uses $f$ - the fraction of critical nodes to total logic blocks. Configuration 2 (a) is used if is $f \leq 1 / 4,2$ (b) is used if $1 / 4 \leq f \leq 1 / 2$ and 2 (c) is used if $1 / 2 \leq f \leq 3 / 4$. Obviously for $3 / 4 \leq f \leq 1$, all the quadrants must be supplied with $\mathrm{V}_{\mathrm{dd}}^{\text {high }}$ and there is no power improvement possible by $\mathrm{V}_{\mathrm{dd}}$ scaling.

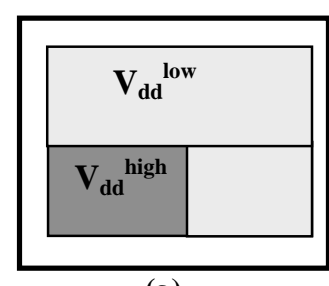

(a)

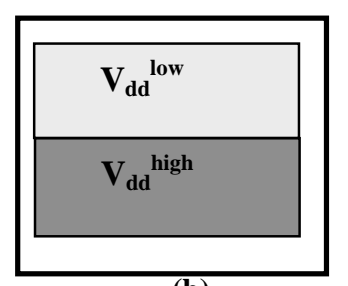

(b)

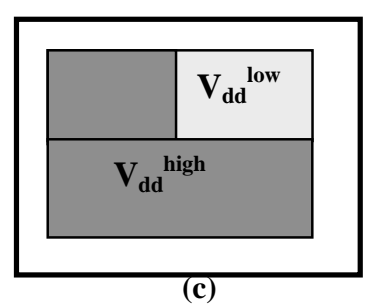

(c)

Figure 2. Proposed Voltage Island configurations in a FPGA

Our placement works as follows: The cluster of critical nodes identified by power driven partitioning algorithm presented in Section 3.2 must be placed in a $\mathrm{V}_{\mathrm{dd}}^{\text {high }}$ region - (determined by $f$ ) otherwise it would mean slowing down the critical path. The power improvement is higher if all non-critical nodes get placed in the $V_{d d}^{\text {low }}$ region although they have a freedom to move to $V_{\mathrm{dd}}^{\text {high }}$ quadrant if it improves the congestion cost.

The placement is based on the simulated annealing implemented in VPR [16]. The linear congestion cost function used in VPR is given by $C_{\text {linear_conj }}=\sum_{i=1}^{N_{\text {nes }}} q(i)\left[\frac{b b_{x}(i)}{C_{a v, x}(i)^{\beta}}+\frac{b b_{y}(i)}{C_{a v, y}(i)^{\beta}}\right]$ where for each net $\mathrm{bb}_{\mathrm{i}}(\mathrm{x})$ and $\mathrm{bb}_{\mathrm{i}}(\mathrm{y})$ denote the horizontal and vertical spans of its bounding box, $\mathrm{q}(\mathrm{i})$ is the compensating factor and $\mathrm{C}_{\mathrm{av}, \mathrm{x}}(\mathrm{i})$ and $\mathrm{C}_{\mathrm{av}, \mathrm{y}}(\mathrm{i})$ are average channel capacitances in the $\mathrm{x}$ and $\mathrm{y}$ directions respectively. Our cost function $C$ is similar to that presented in [16] and is given as:

$$
\Delta C=\Delta C_{\text {linear_conj }}+\alpha \Delta \text { matched }(j)+\gamma(1-\text { matched }(j))
$$

where matched(j) returns 1 if the $j^{\text {th }}$ logic block is placed in its matching voltage quadrant and 0 if not. $\Delta$ matched is the difference between matched(j) in the previous placement and matched(j) in the present placement and penalizes a move that brings a block from matched quadrant to unmatched quadrant; (1-matched(j)) penalizes a move that moves a block in unmatched quadrant to another location in the unmatched quadrant; $\alpha, \gamma$ are appropriate constants and $\gamma>\alpha$. 
For FPGAs it is common practice to pack the LUTs into logic blocks. We used TVpack [16] to pack LUTs with clusters of size 4 and 10 inputs per cluster option. After packing, the power driven partitioner partitions the logic blocks for $\mathrm{V}_{\mathrm{dd}}{ }^{\text {high }}$ and $\mathrm{V}_{\mathrm{dd}}{ }^{\text {low }}$ regions. For apex 2 benchmark the characteristics are: the smallest FPGA size is $23 \times 23$ logic array, out of 485 clusters there are 66 critical logic blocks and $f$ is $12 \%$. The placement is of type shown in Figure 2(a) and the placed circuit is shown in Figure 3.

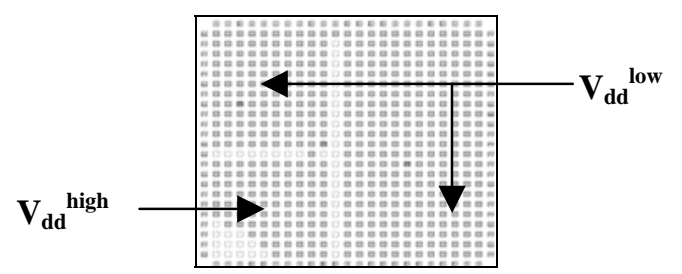

Figure 3. Constrained Placement of LUTs clusters of apex2

Ten MCNC benchmarks were clustered and Table 3 shows their total number of logic blocks, primary inputs (PI) and outputs (PO), \% $f$ and the FPGA size $D_{X} \times D_{Y}$. Wire length (WL) and channel width (CW) are shown for unconstrained placement vs. power driven partitioning and region-constrained placement. We also report the number of non-critical blocks in the $\mathrm{V}_{\mathrm{dd}}$ low region and the power improvement over unconstrained partitioning. The results show that it is possible to get an average power improvement of $48 \%$ with $23 \%$ penalty in channel width and $26 \%$ penalty in wire length.

Table 3. Placement and Routing results for MCNC benchmarks

\begin{tabular}{|c|c|c|c|c|c|c|c|c|c|c|c|c|}
\hline & \multirow{2}{*}{\multicolumn{2}{|c|}{ Unconstrained }} & \multirow{2}{*}{\multicolumn{4}{|c|}{ Constrained-Power Driven }} \\
\hline & & & & & & & & & & & & \\
\hline Circuits & \begin{tabular}{|c|} 
Logic \\
Blocks
\end{tabular} & $\begin{array}{c}\text { Crit } \\
\text { Block }\end{array}$ & $D x=D y$ & $\% f$ & $\mathrm{PI}$ & $\mathrm{PO}$ & WL & $\mathrm{CW}$ & WL & CW & $\begin{array}{l}\text { Blocks } \\
\text { in } V_{\text {low }}\end{array}$ & $\% \operatorname{lmp}$ \\
\hline alu4 & 389 & 51 & 20 & 13 & 14 & 8 & 20262 & 33 & 22151 & 37 & 289 & 46 \\
\hline apex2 & 485 & 66 & 23 & 12 & 38 & 3 & 31738 & 40 & 37348 & 47 & 385 & 49 \\
\hline apex4 & 334 & 77 & 19 & 21 & 9 & 19 & 20317 & 40 & 23383 & 48 & 247 & 46 \\
\hline des & 415 & 47 & 32 & 5 & 256 & 245 & 25199 & 25 & 44587 & 41 & 357 & 53 \\
\hline ex1010 & 1201 & 250 & 5 & 20 & 10 & 1 & 16 & 43 & 104744 & 69 & 908 & 47 \\
\hline ex5p & 280 & 22 & 17 & 8 & 8 & 63 & 17572 & 41 & 19625 & 47 & 213 & 47 \\
\hline misex3 & 362 & 57 & 20 & 14 & 14 & 14 & 21650 & 36 & 24606 & 39 & 281 & 48 \\
\hline pdc & 1190 & 141 & 35 & 12 & 16 & 40 & 98312 & 61 & 116923 & 71 & 911 & 48 \\
\hline seq & 448 & 94 & 22 & 19 & 41 & 35 & 27904 & 39 & 35767 & 48 & 342 & 47 \\
\hline spla & 955 & 133 & 31 & 14 & 16 & 46 & 71330 & 57 & 86536 & 66 & 717 & 47 \\
\hline \multicolumn{7}{|l|}{ Average } & 40720 & 42 & 51567 & 51 & 465 & 48 \\
\hline \multicolumn{5}{|c|}{ Avg. Penalty fo } & ire & th & $26.64 \%$ & \multicolumn{3}{|c|}{ Channel width } & $23.61 \%$ & \\
\hline
\end{tabular}




\section{Conclusions}

In this paper, we presented a power optimization technique for FPGA-based systems. Our proposed approach aims to create opportunities for voltage scaling by grouping nodes in a LUT-level netlist into clusters. The partitioning approach targets to exploit the maximum flexibility in timing constraints and convert it into voltage scaling. We developed a partitioning algorithm to perform this task. Within the general framework of our algorithm resource constraints can be resolved. Our experimental results reveal that it is indeed effective. Based on the high-level power improvement estimation, we did a constrained placement of the clusters onto voltage islands in a single FPGA and showed power improvement vs. penalty in wire length and channel width increase. Hence possible tradeoffs between cost function and power improvement is evident. An immediate extension to our work is to evaluate and actually compare delay after physical design due to constrained placement and area-power tradeoffs due to the presence of level converters.

\section{References}

1. Boemo, E.I., et al., eds. Some Notes on Power Management on FPGA-based Systems. Lecture Notes in Computer Science. Vol. 975. 1995, Springer-Verlag: Berlin. 149--157.

2. Chen, C., T. Hwang, and C.L. Liu. Low Power FPGA design - A Re-engineering Approach. in Design Automation Conference. 1997.

3. Sutter, G., et al. FSM Decomposition for Low Power in FPGA. in Design Automation Conference. 1998

4. Chen, D., J. Cong, and Y. Fan. Low Power High-Level Synthesis for FPGA Architectures. in International Symposium on Low Power Electronic Design. 2003.

5. Lamoureux, J. and S.J.E. Wilton. On the Interaction Between Power-Aware FPGA CAD Algorithms. in International Conference on Computer Aided Design. 2003.

6. Brasen, D.R. and G. Saucier, Using Cone Structures for Circuit Partitioning into FPGA Packages. IEEE Transactions on CAD of Integrated Circuits and Systems, 1998. 17(7): p. 592--600.

7. Chan, P.K., M.D.F. Schlag, and J.Y. Zien. Spectral-Based Multi-Way FPGA Partitioning. in International Symposium on Field Programmable Gate Arrays. 1995.

8. Chang, D. and M. Marek-Sadowska, Partitioning Sequential Circuits on Dynamically Reconfigurable FPGAs. IEEE Transactions on Computers, 1999. 48(6): p. 565--578.

9. Liu, H. and D.F. Wong. Circuit Partitioning for Dynamically Reconfigurable FPGAs. in International Symposium on Field Programmable Gate Arrays. 1999.

10. Govil, K., E. Chan, and H. Wasserman. Comparing Algorithms for Dynamic Speed Setting of a Low Power CPU. in MOBICON. 1995.

11. Iyer, A. and D. Marculescu. Power Efficiency of Voltage Scaling in Multiple Clock, Multiple Voltage Cores. in International Conference on Computer Aided Design. 2002.

12. Yeh, C., et al. Gate-Level design Exploiting Dual Supply Voltages for Power-Driven Applications. in Design Automation Conference. 1999.

13. Simunic, T., et al. Dynamic Voltage Scaling and Power Management for Portable Systems. in Design Automation Conference. 2001.

14. Chen, D., et al. Low-Power Technology Mapping for FPGA Architectures with Dual Supply Voltage. in International Symposium on Field-Programmable Gate Arrays. 2004.

15. Li, F., et al. Low-Power FPGA Using Pre-Defined Dual-Vdd/Dual-Vt Fabrics. in International Symposium on Field-Programmable Gate Arrays. 2004.

16. Betz, V., J. Rose, and A. Marquardt, Architecture and CAD for Deep-Submicron FPGAs. Kluwer Academic Publishers, Feb 1999. 